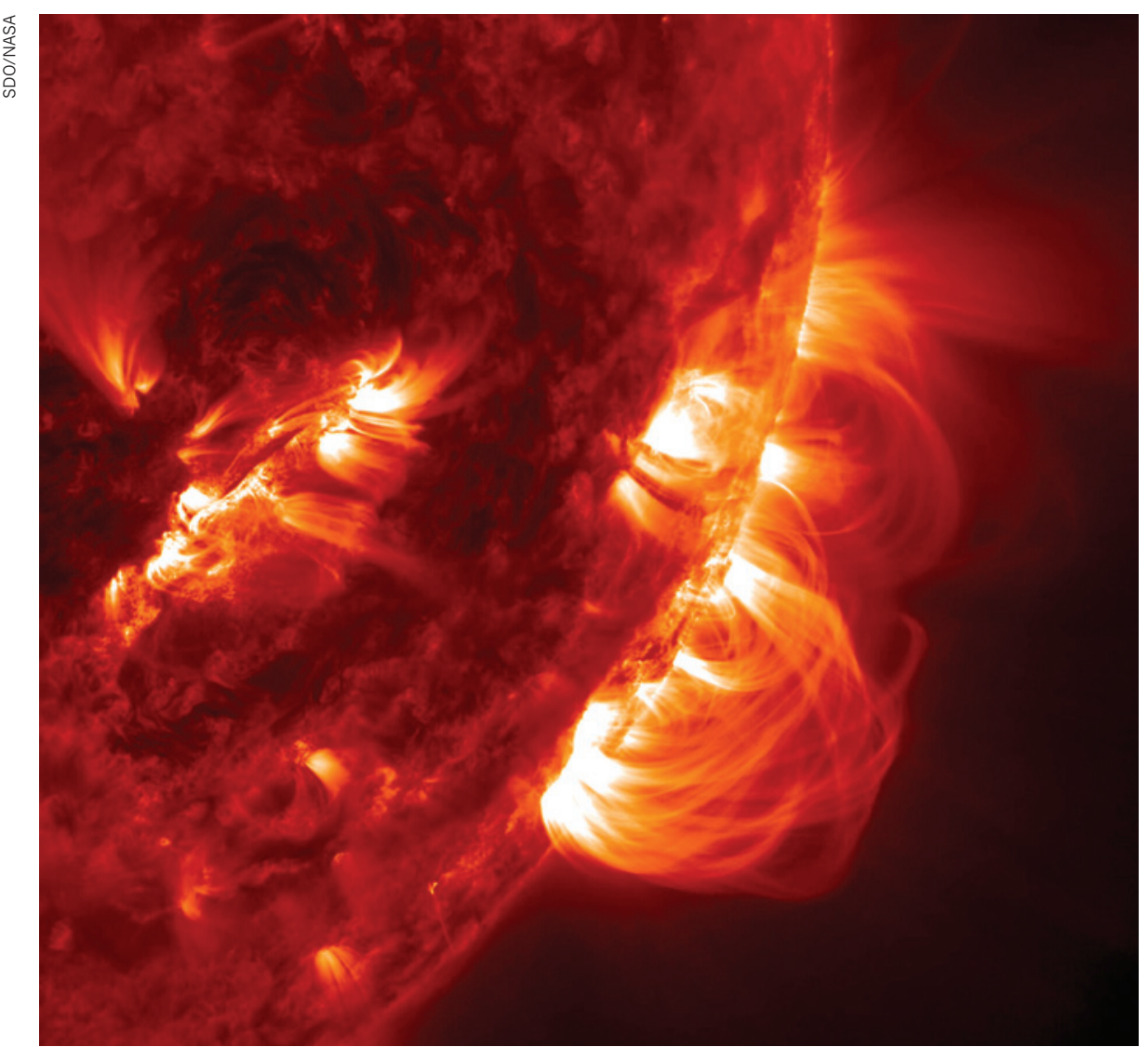

A type of turbulence simulated in a lab experiment could help to explain why the Sun's corona is so hot.

\title{
PHYSICS
}

\section{Lab astrophysics aims for the stars}

\section{Earth experiments deployed to understand data from space.}

\section{BY EUGENIE SAMUEL REICH}

$\mathrm{T}$ he giant orange magnets were built decades ago to confine hydrogen nuclei in the quest for fusion energy. But since 1998, Jan Egedal, a plasma physicist at the Massachusetts Institute of Technology in Cambridge, has used the magnets, from a massive, doughnut-shaped tokamak, to simulate magnetic fields in the thin wind of charged particles streaming from the Sun. Egedal hopes to learn how the solar wind transfers energy.

In the past, 'laboratory astrophysics' experiments such as Egedal's - efforts to mimic the behaviour of space plasmas and other astrophysical phenomena - have had to piggyback on the apparatus of energy research or fundamental physics. Now, practitioners are trying to enshrine the field as a discipline in its own right, with dedicated funding and equipment.
In June, the American Astronomical Society (AAS) in Washington DC created its first new division in 30 years, dedicated to lab astrophysics. The American Physical Society's plasmaphysics meeting last month in Providence, Rhode Island, saw an unprecedented number of lab-astrophysics sessions. And some NASA scientists are saying that the agency should devote a small amount of funding from every space mission to lab astrophysics. "There's an increasing number of papers on lab astrophysics," says Fred Skiff, a plasma physicist at the University of Iowa in Iowa City, who chaired the physical-society meeting. "It's come of age."

One goal is to make the most of the data streaming in from spacecraft. Egedal, for example, is exploring questions posed by two solarwind missions: the European Space Agency's Cluster and NASA's Wind. "We're in the heyday of Solar System spacecraft, and that's fuelled the growth of lab-astrophysics experiments," he explains. Farid Salama, a lab astrophysicist at NASA's Ames Research Center in Moffett Field, California, says that the work is also attractive in times of tight budgets, because the cost of experiments tends to be hundreds of thousands of dollars, compared with hundreds of millions for space missions.

Significant results are emerging. Last month, Skiff and his colleagues described the first lab measurement of astrophysical turbulence in which two magnetic waves collide to generate a third one - a phenomenon that has been invoked to explain why the Sun's atmosphere, or corona, is thousands of time hotter than its surface and how massive amounts of energy move between galaxies. Using the Large Plasma Device, a 21-metre-long plasma generator at the University of California, Los Angeles, Skiff made two of these 'Alfvén' waves, collided them and mapped the daughter wave, confirming the mechanism (G. G. Howes et al. Phys. Rev. Lett. Preprint at http://arxiv.org/abs/1210.4568; 2012). "This is the first clear lab demo of something that is a cornerstone of theory," says Amitava Bhattacharjee, a theoretical astrophysicist at Princeton University in New Jersey. The experiment cost less than US\$100,000.

NASA has always funded some lab astrophysics, but Salama says that the agency should consider doing so as a matter of course for all space missions. A good first candidate for this funding model, he says, would be work connected with the Herschel Space Observatory, a European mission with NASA involvement that is collecting infrared spectra from thousands of different molecules in Galactic clouds of dust and gas. Many of the molecules have yet to be identified, but researchers could pin them down by synthesizing candidates and checking their spectra in the lab. Daniel Savin, an astrochemist at Columbia University in New York who was involved in the creation of the AAS lab-astrophysics division, says that theoretical work in lab astrophysics is also needed to set priorities for which experiments to do. For example, synthesizing every possible molecule that might be observed in space is not financially feasible. Models of cloud chemistry could guide the work by indicating which are the most important.

Dedicated funding for lab astrophysics would be good news for Egedal, who is beginning to outgrow his current apparatus. His group has predicted the existence of a plasma regime in which electrons can be unexpectedly and powerfully energized, a previously unexplained effect seen by spacecraft observing the solar wind. But to explore the mechanism, he needs to do experiments using a larger volume of plasma and higher magnetic fields (J. Egedal et al. Nature Phys. 8, 321-324; 2012). If lab astrophysics establishes itself as a worthy discipline, he might be able to graduate from his hand-me-down tokamak, he says. "I want to build a new one." - 